\title{
Instrumentación mecanizada en molares primarios: ¿opción o necesidad?
}

\author{
\begin{tabular}{|r} 
Monique Lopes Reis ${ }^{1}$; \\
Patrícia Maria Coelho \\
Milena Carvalho \\
Rogério Vieira Silva \\
; \\
Thayse Pithon Quadros Ravazzi
\end{tabular}
}

\section{Resumen}

Objetivo: Analizar el tiempo de instrumentación radicular en molares primarios artificiales por la técnica manual y mecanizada y comparar a través de radiografía digital la calidad de la obturación de los canales radiculares. Materiales y métodos: La muestra fue de 65 dientes, siendo 5 destinados a la calibración del estudio (G1), 30 en la instrumentación mecanizada reciprocante (G2) y 30 en la instrumentación manual (G3). El tiempo de instrumentación fue cronometrado. Después de la instrumentación, se realizó la obturación de los canales radiculares en ambos grupos. Para el análisis de la calidad de la obturación, se utilizaron radiografías digitales. La prueba D 'Agostino fue utilizada, y los datos presentaron distri- bución no normal. Para evaluar el tiempo de instrumentación y la calidad de la obturación se utilizó el test estadístico Mann-whitney. El nivel de significancia establecido fue del 5\%. Resultados: El tiempo de instrumentación por la técnica mecanizada fue más rápido que la instrumentación manual ( $p<0,05)$, y el grado de la calidad de la obturación se mostró más eficaz en los dientes instrumentados por la técnica mecanizada $(p<0,05)$. Conclusión: La técnica de instrumentación mecanizada se mostró más eficaz en la reducción del tiempo y en el grado de llenado de los canales radiculares, siendo que su uso proporcionó ventajas en la rutina clínica pediátrica.

Palabras Clave: Odontopediatría, endodoncia, obturación del conducto radicular.

\footnotetext{
1. Graduanda en Odontologia, Faculdade Independente do Nordeste, Vitoria da Conquista, Brasil.

2. Professora Titular de la Disciplina Odontopediatria de la Faculdade Independente do Nordeste, Vitoria da Conquista, Brasil.

3. Professora Titular de la Disciplina Odontopediatria de la Faculdade Independente do Nordeste, Vitoria da Conquista, Brasil.

4. Professor Titular de la Disciplina Odontológica II de endodontia de la Faculdade Independente do Nordeste, Vitoria da Conquista, Brasil.

5. Professora Titular de la Disciplina Estagio Supervisionado IV de endodontia de la Faculdade Independente do Nordeste, Vitoria da Conquista, Brasil.
} 
Artigo Original

\section{Instrumentação mecanizada em molares primarios: ¿opção ou necessidade?}

\section{Resumo}

Objetivo: Analisar o tempo de instrumentação radicular em molares decíduos artificiais pela técnica manual e mecanizada e comparar, através de radiografia digital, a qualidade da obturação dos canais radiculares. Material e Métodos: A amostra consistiu de 65 dentes, sendo 5 destinados a calibração do estudo (G1), 30 na instrumentação mecanizada reciprocante (G2) e 30 na instrumentação manual (G3). O tempo de instrumentação foi cronometrado. Após a instrumentação, foi realizada a obturação dos canais radiculares em ambos os grupos. A análise da qualidade da obturação, foi medida por meio de radiografias digitais. $\mathrm{O}$ teste $\mathrm{D}^{\prime}$ Agostino foi utilizado, e os dados apresentaram distribuição não normal. Para avaliar o tempo de instrumentação e a qualidade da obturação foi utilizado o teste estatístico Mann-whitney. O nível de significância estabelecido foi 5\%. Resultados: O tempo de instrumentação pela técnica mecanizada foi mais rápido que a instrumentação manual $(p<0,05)$, e o grau da qualidade da obturação mostrou-se mais eficaz nos dentes instrumentados pela técnica mecanizada $(\mathrm{p}<0,05)$. Conclusão: $\mathrm{A}$ técnica de instrumentação mecanizada mostrou-se mais eficaz na redução do tempo e no grau de preenchimento dos canais radiculares, sendo que seu uso proporcionou vantagens na rotina clínica pediátrica.

Palavras-Chaves: Odontopediatria, endodôntia, obturação do canal radicular.

\section{Original article}

\section{Mechanized instrumentation in deciduous molars: option or necessity?}

\begin{abstract}
Aim: To analyze instrumentation time in artificial deciduous molars by manual and mechanized technique and to compare the quality of root canal filling using digital radiography. Material and Methods: The sample consisted of 65 teeth, of which 5 were used for calibration of the
\end{abstract}

study (G1), 30 for reciprocating mechanized instrumentation (G2) and 30 for manual instrumentation (G3). The instrumentation time was timed. After the instrumentation, the root canals were obturated in both groups. In order to analyze the quality of the obturation, digital radiographs were used. The D 'Agostino test was used, and the data presented a non-normal distribution. The Mann-Whitney test was used to evaluate the 
instrumentation time and the quality of the obturation. The level of significance was set at $5 \%$. Results: Mechanized instrumentation time was faster than manual instrumentation $(\mathrm{p}<0.05)$, and the degree of obturation quality was more effective in the teeth instrumented by the mechanized technique $(p<0.05)$. Conclusion: The mechanized

\section{Introducción}

La odontopediatría tiene como principio mantener la integridad y función de la dentición primaria hasta su exfoliación fisiológica. En algunos casos de dientes con necrosis pulpar, el tratamiento endodóntico debe ser realizado. Esto evita la pérdida precoz de los dientes primarios que, a su vez, puede causar alteraciones en la cronología de erupción del permanente, contribuyendo a problemas en la masticación, fonética y estética de los niños. ${ }^{1}$ La terapia en dientes primarios a veces no es llevada en rigor, justificada por el complicado comportamiento infantil y también por diversas modificaciones anatómicas de los dientes primarios. Es importante simplificar y optimizar el tratamiento en dientes. ${ }^{2,3}$

La instrumentación del sistema de canales radiculares en dientes primarios ha sido exitosa con el auxilio de instrumentos reciprocantes, que optimizan el tiempo clínico en la atención y demuestra gran aceptación en la literatura actual. Sin embargo, el tratamiento debe ser conducido buscando siempre la efectividad de la preparación biomecánica, la limpieza y la obturación del sistema de canales. ${ }^{1}$

La instrumentación mecánica y manual no muestran diferencias significativas en la capacidad de limpieza, pero la instrumentación reci- instrumentation technique proved to be more effective in reducing the time and degree of filling of the root canals, and its use provided advantages in the pediatric clinical routine.

Keywords: Pediatric dentistry, endodontics, root canal obturation.

procante demostró ser más eficaz en la reducción del tiempo, pues se utiliza una sola lima con la técnica corona ápice, capaz de alcanzar toda la longitud del canal radicular del diente primário, incluso en canales curvos, además de causar poco o ningún desplazamiento en la región apical. Una vez que el mantenimiento de la trayectoria original del canal es esencial para asegurar la integridad del germen sucesor permanente. ${ }^{4}$

La eficacia de la terapia está directamente relacionada con los principios biológicos, donde las etapas de procedimiento consisten en: reducción microbiana del sistema de canales radiculares con la preparación biomecánica, limpieza y desinfección con la remoción de detritos, modelado y sellado del canal radicular, seguida de la preservación del caso. ${ }^{1}$ En este contexto, el uso de un material obturador adecuado es esencial en el sellado del canal, pues impide la penetración de microorganismos y sus toxinas, permite la reparación periapical e impide la reinfección.,

El material de relleno del conducto radicular de dientes primarios debe presentar requisitos básicos como: ser reabsorbible, no causar lesiones a los tejidos periodontales y sucesor permanente, presentar propiedades antimicrobianas, posibilitar condiciones de reparación, así como llenar de forma satisfactoria a las paredes del conducto radicular, ser radiopaco y no generar coloración al diente. ${ }^{7,8}$ 
Por lo tanto, el objetivo de esta investigación fue comparar el tiempo clínico de instrumentación manual y mecánica en molares primarios artificiales y analizar el grado de llenado de la obturación de los dientes instrumentados, tanto por la técnica mecánica como por la técnica manual, en radiografías digitales.

\section{Materiales y metodos}

La parte experimental del estudio fue realizada en el laboratorio de la clínica escuela de odontología de la Facultad Independiente del Nordeste (FAINOR). El número de muestra fue justificado a partir del cálculo muestral utilizando el software GPower (Heinrich-Heine-Universität Düsseldorf), de forma que se utilizaron un total de 65 molares superiores primarios artificiales con pulpa (DENARTE, São Paulo, SP, Brasil). Siendo que 5 dientes se utilizaron para la calibración del estudio (G1). A partir de eso los dientes fueron divididos en dos grupos: $\mathrm{G} 2$ instrumentados por la técnica reciprocante $(n=30)$ y G3 instrumentados por la técnica manual $(\mathrm{n}=30)$. Inicialmente todos los dientes fueron radiografiados y excluidos de la muestra los que presentaban cualquier tipo de impedimento que interfiriera la instrumentación. Un operador con amplia experiencia en el sistema realizó la instrumentación. Fijados a un torno, realizó el acceso endodóntico en los dientes con la fresa \#1014 (KG SORENSEN, São Paulo, SP, Brasil), y fresa Endo Z (MICRODONT, São Paulo, SP, Brasil). Insertó una lima tipo 15 k-file (Maillefer Intruments, Ballaigues, Suiza) hasta el foramen apical, obteniendo de esa manera la longitud real del diente (CRD), por el método visual, y la longitud de trabajo $1 \mathrm{~mm}$ por debajo del CRD. Se utilizó el instrumento Reciproc R25 acoplado al motor X-Smart Plus (Maillefer Instruments, Ballaigues, Suiza), siguiendo

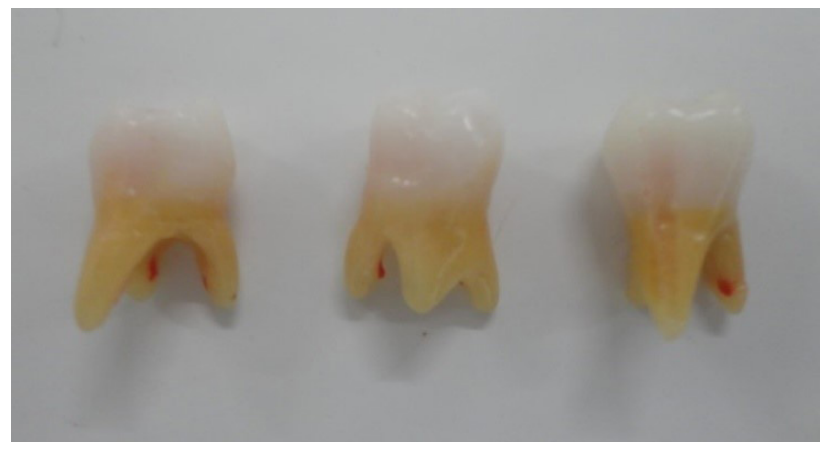

Figura 1. Molares superiores primarios artificiales con pulpa radicular.

las instrucciones del fabricante, siendo que cada dos dientes instrumentados, el instrumento era sustituido. En la instrumentación el tiempo fue cronometrado. Los dientes fueron irrigados con solución fisiológica (Arboreto, Juiz de Fora, MG, Brasil) durante el procedimiento, seguidos de secado con cono de papel absorbente \#25 (Dentsply, Petrópolis, RJ, Brasil). Después de la instrumentación del G2, se procedió a la instrumentación manual del G3, con limas manuales k-file \#15, \#20 e \#25 (Maillefer Dentsply, Ballaigues, Suiça) por la técnica Manual de Escalonamiento "CrownDown". Después de concluido todo el procedimiento de instrumentación, los dientes fueron ob-

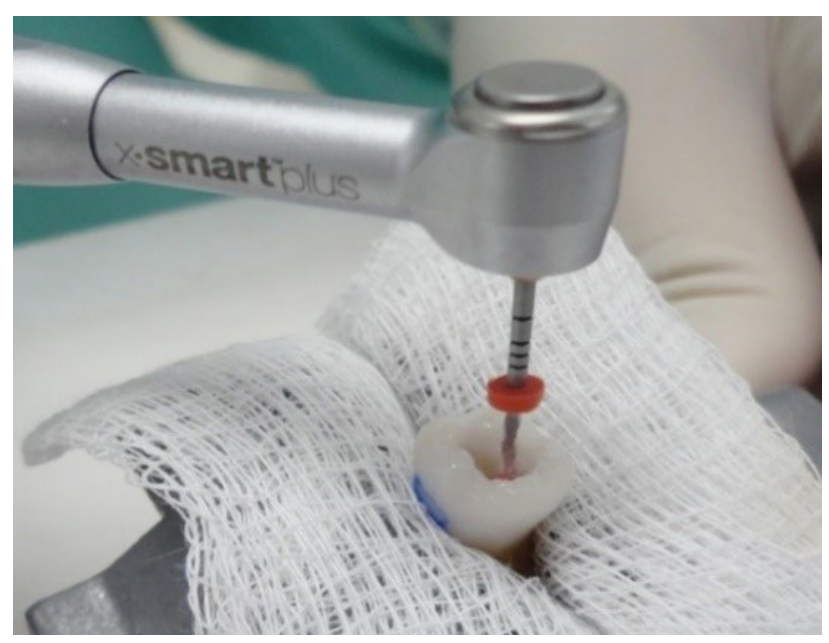

Figura 2. Instrumentación mecanizada (flecha roja indica el equipo utilizado) con instrumento Reciproc \# 25 (flecha amarilla). 

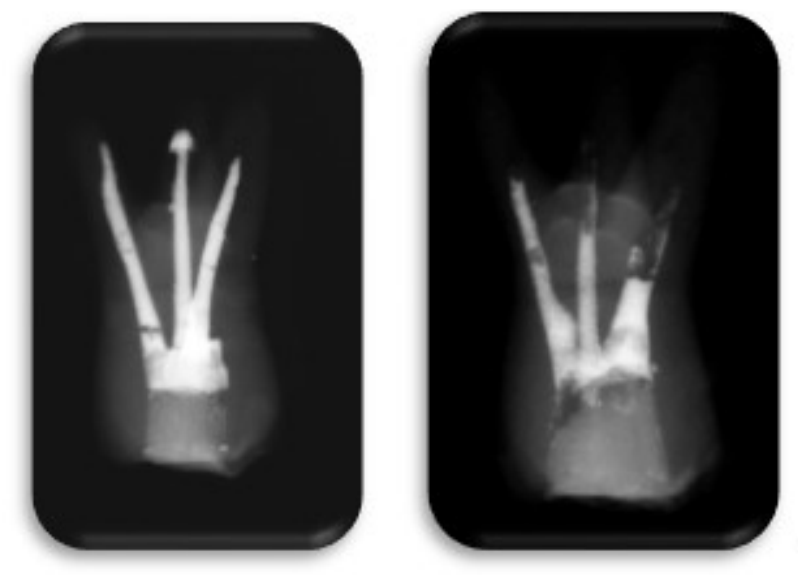

Figura 3. Radiografía periapical digital final en incidencia ortorradial y mesioradial por la instrumentación mecanizada.

turados con la utilización de la pasta Guedes-Pinto, siguiendo rigurosamente la proporción propuesta por Mello-Moura, de 1cm de Ricfocort (Medley, Campinas, SP, Brasil), $1 \mathrm{~cm}$ de un tubo anestésico con iodoformo (Biodinámica (Biodinámica, Paraná, Brasil), y dos gotas de paramonoclorofenol alcanforado (PMCC) (Biodinámica, Paraná, Brasil), que fueron insertados en el conducto radicular de una sola forma: Lima k-file \#25 (Maillefer Instruments, Ballaigues, Suiza), en ambos grupos. Para el análisis de la calidad de la obturación, se utilizaron radiografías digitales. En el análisis estadístico se utilizaron el programa Bioestat 5.3. Se aplicó el ensayo D 'Agostino fue utilizado, y los datos presentaron distribución no normal. Para evaluar el tiempo de instrumentación y la calidad de la obturación se utilizó el ensayo estadístico Mann-whitney. El nivel de significancia establecido fue del $5 \%$.

\section{Muestra y grupos}

Tiempo de instrumentación:

- G2 (instrumentación mecanizada): 30 dientes instrumentados por la técnica mecanizada con instrumento recíproco R25.
- G3 (instrumentación manual): 30 dientes instrumentados por la técnica manual con limas manuales k-file \# 15, \# 20 y \# 25 .

En ambos grupos, el tiempo fue cronometrado por dos personas, siendo al principio de la instrumentación y pausado en el momento de la irrigación y cambio de limas.

\section{Obturación de los dientes:}

La obturación de los canales radiculares en ambos grupos se realizó con la pasta obturadora descrita anteriormente, en la que fue insertada por lima \# 25 , con los movimientos horarios de $1 / 4$ de vuelta por tres veces, en cada conducto radicular.

\section{Evaluador}

Por tratarse de un estudio ciego, las imágenes fueron dirigidas a dos radiólogos que desconocían la muestra, debidamente el Kappa, que siguió la siguiente escala:

- 1 Imágenes de dientes completamente sin material obturador en los conductos radiculares;

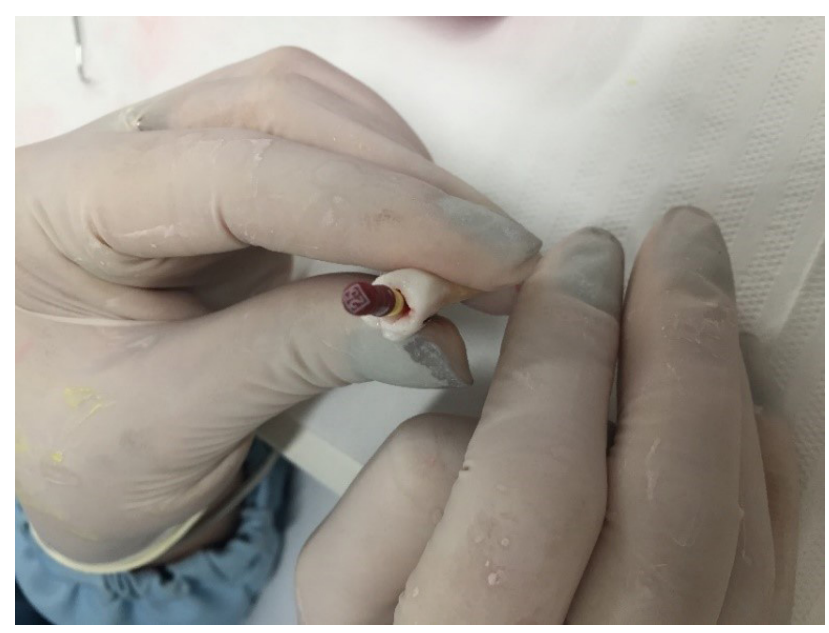

Figura 4. Instrumentación manual (flecha roja indica el instrumento utilizado) con instrumento manual $K$ - file \#25. 
- 2 Imágenes de dientes con material presente sólo en la cámara pulpar;

- 3 Imágenes de dientes con canales parcialmente rellenos;

- 4 Imágenes de dientes con canales completamente rellenos.

\section{Análisis de los datos}

El ensayo D'Agostino se aplicó para verificar la normalidad (muestra no normal). El nivel de significancia fue establecido en el 5\%, y para evaluar la diferencia entre los grupos de tiempo de instrumentación y de obturación se utilizó el ensayo Mann-whitney para ambos.

\section{Resultados}

Al verificarse la diferencia entre los grupos, el grupo 2, en relación al tiempo de instrumentación manual, presentó valor mediano de 2.40 ", valores mínimo y máximo entre 2.2 y 3.04 res-

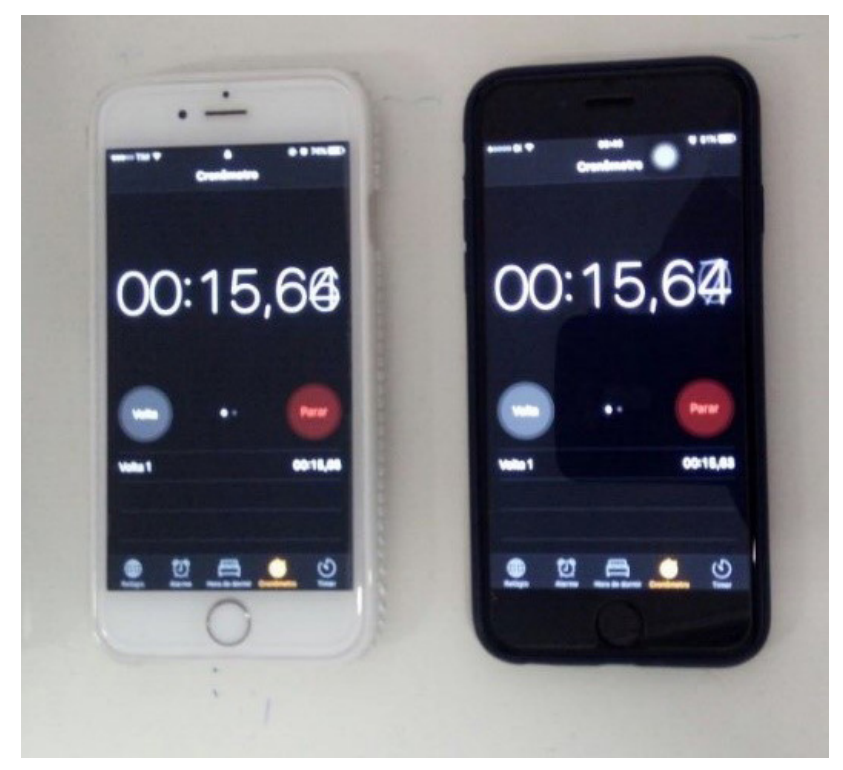

Figura 5. Cronometrando el tiempo de la instrumentación manual el mecanizada.

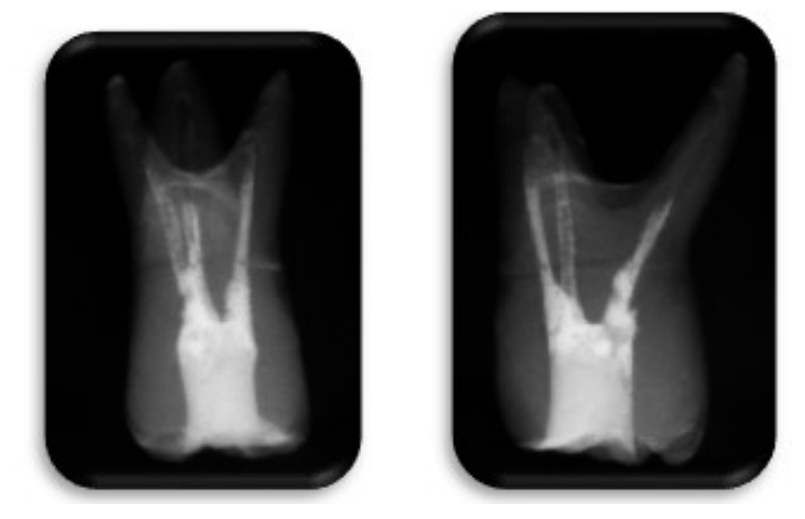

Figura 6. Radiografía periapical digital final en incidencia ortorradial y mesioradial por la técnica de instrumentación manual.

pectivamente. El grupo 3, en relación al tiempo de instrumentación mecânica, presentó valor mediano de 1.50 ", valores mínimo y máximo entre 1.07 y 2.12 respectivamente. Ambos grupos del tiempo de instrumentación se obtuvieron con relevancia estadística $(\mathrm{p}<0.05)$. (Tabla 1$)$

Al analizarse los grupos en relación a la incidencia radiográfica de obturación de los canales en relación a la técnica de instrumentación, el grupo 2, con instrumentación manual, presentó un valor mediano 3 (canales parcialmente rellenados), valores mínimo y máximo entre 2 y 3 . En el grupo 3, con instrumentación mecánica, se obtuvo un valor mediano 3 (canales parcialmente rellenados), valores mínimo y máximo entre 3 y 4 y tam-

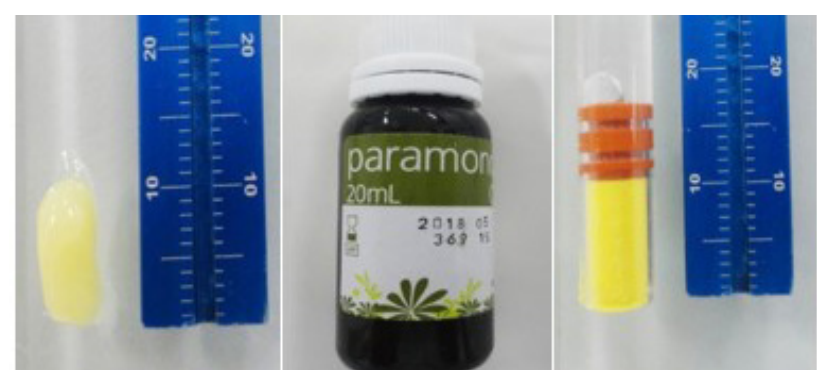

Figura 7. Cronometrando el tiempo de la instrumentación manual el mecanizada. 


\begin{tabular}{|c|c|c|}
\hline Manual & Mecanizada & ${\text { Valor de } \mathbf{p}^{*}}_{\text {Mediana (Mínima- Maximo) }}$ \\
\hline $2^{\mathrm{a}} \cdot 40^{\mathrm{b}}(2.2-3.04)$ & Mediana (Mínima- Maximo) & $<0,05$ \\
\hline
\end{tabular}

*Valor de p obtido pelo teste Mann-whitney

a: tiempo en minutos de la instrumentación manual; b: tiempo en segundos de la instrumentación manual c: tiempo en minuto en la instrumentación mecanizada; d: tiempo en segundos de la instrumentación mecanizada

Tabla 1. Diferencia entre el tiempo de la instrumentación manual y mecanizada en molares decíduos.

bién hubo diferencia estadísticamente significativa entre los grupos $(p<0,05)$. (Tabla 2$)$.

\section{Discusión}

Debido a la complicación para obtener dientes primarios naturales que puedan ser utilizados en investigaciones, se incentivó la formulación de un modelo pedagógico de dientes primarios artificiales, teniendo su anatomía detallada y radiopaca, siendo sus ejemplares compuestos de resinas sintéticas, representado el aspecto de los dientes primarios humanos naturales, ${ }^{10} \mathrm{o}$ que justificó su uso en la búsqueda.

De acuerdo con Guedes-Pinto y Santos (2010), ${ }^{11}$ mantener los dientes primarios en el arco dental hasta la exfoliación fisiológica permite la erupción correcta de la dentición permanente, favoreciendo el crecimiento maxilo-mandibular de forma más armónica. La indicación de tratamiento endodóntico se hace necesario en casos especificos de necrosis pulpar sin envolvimiento de la furca y debe ser realizado de forma eficiente en todas sus etapas clínicas, ${ }^{12}$ lo que hizo relevante este estudio.

Uno de los factores importantes en el tratamiento endodóntico en la Odontopediatría es proporcionar una instrumentación adecuada en un menor tiempo clínico posible. En esta investigación, se optó por la instrumentación de las dos técnicas, manual y mecanizada, para observar cuál de las técnicas se mostraría más eficaz en la reducción del tiempo de instrumentación de los canales radiculares.

Silva et al. (2004), ${ }^{13}$ Kummer et al. $(2008)^{14}$ y Aragatina et al. (2010) ${ }^{15}$ han concluido que la instrumentación reciprocante es estadísticamente mejor, comparado con la instrumentación manual, relativo al tiempo de la instrumentación..$^{13-15}$ Lo que está

\begin{tabular}{|c|c|c|}
\hline $\begin{array}{c}\text { Manual } \\
\text { Mediana (Mínima- Maximo) }\end{array}$ & Mecanizada & ${\text { Valor de } \mathbf{p}^{*}}^{\text {Mediana (Mínima- Maximo) }}$ \\
\hline $3^{\mathrm{a}}\left(2^{\mathrm{b}}-3\right)$ & $3^{\mathrm{a}}\left(3-4^{\mathrm{c}}\right)$ & $<0,05$ \\
\hline
\end{tabular}

*Valor de p obtido pelo teste Mann-whitney

a: canales parcialmente rellenos; b: material presente sólo en la cámara pulpar; c: canales completamente rellenos.

Tabla 2. Diferencia de la obturación de molares decimos instrumentados manual y mecanicamente. 
de acuerdo con diversos autores, que observaron que la optimización del tratamiento endodóntico en dientes primarios es importante, pues mejora la calidad del mismo y disminuye el tiempo clínico. 16-17

Mello-Moura et al. $(2013)^{2}$, afirmaron en su trabajo que la mecanización de la preparación radicular en la terapia de dientes permanentes ya es una realidad, proporcionando menor tiempo de tratamiento, sin perder eficiencia y reduciendo también el esfuerzo clínico y físico del profesional. Por estas razones, estos sistemas mecanizados pueden y deben ser adecuados para la preparación de la terapia pulpar en dientes. ${ }^{2} \mathrm{~A}$ través de la introducción de técnicas que determinan simultáneamente eficiencia, economía y adecuación al comportamiento del niño de temprana edad, el trabajo se ha tornado fácilmente accesible al dentista clínico. ${ }^{12}$

Entre los diversos tipos de pastas obturadoras que se encuentran en el mercado, pasta básicamente compuesta de óxido de zinc y eugenol, con la adición de antibióticos de amplio espectro y tetraciclina cloranfenicol (CTZ), pasta de óxido de zinc y eugenol (OZE), y la base de hidróxido de calcio $[\mathrm{Ca}(\mathrm{OH}) 2],{ }^{17}$ la investigación se optó por la pasta Guedes-Pinto compuesta por PMCC, Iodofórmio y Ricfocort ${ }^{\circledR}$, en sus debidas proporciones, el uso de la misma se justifica por Mello-Moura et al. (2007), ${ }^{7}$ por presentar propiedades antimicrobianas, antisépticas, radiopacidad, capacidad de reabsorción, posibilitando la estimulación biológica, siendo también antiinflamatorio, caracterizando asociación corticosteroide-antibiótica. ${ }^{7}$ Es importante resaltar también que en estudios hechos por Kramer et al. (2000) ${ }^{18}$ y Bruscos et al. (2000) ${ }^{19}$, fue concluido que es el material obturador más utilizado en Odontopediatría en las facultades de Odontología de Brasil. ${ }^{20}$
En un estudio realizado por Guedes-Pinto $(2012),{ }^{20}$ basado en acompañamiento radiográfico y clínico durante dos años con muestra de 45 dientes primarios obturados con la pasta que lleva su nombre, se puede concluir el 97,7\% de éxito, siendo que los dientes con fístula y/o movilidad patológica obtuvieron neoformación en 60 días, y la pasta extravasada en 30 días era totalmente reabsorbible, con una indicación comprobada para el tratamiento de los dientes primarios comprometidos. ${ }^{20}$

A pesar de Souza et al. (2005), ${ }^{3}$ después de la evaluación de la introducción del material obturador, concluir que el léntulo y la lima son efectivos para introducir pasta obturadora hasta la longitud de trabajo, con relación a la lateralidad, el léntulo se mostró superior a la lima. En contrapartida, hubo una extravasación mayor del material cuando éste era introducido con el léntulo, ${ }^{3}$ no siendo encontrada diferencia estadísticamente significante que favoreciera a cualquiera de los grupos.

De este modo, en esta investigación se utilizó la lima tipo k-file para ambas obturaciones en las dos técnicas de instrumentación, en la que no justifica la utilización de léntulo para inserción de material obturador en la dentición primaria, además de ella aún presentar un costo adicional, mayor riesgo de fractura y mayor extravasación del material obturador cuando se comparan con la lima tipo k-file ${ }^{3}$.

\section{Conclusión}

A partir de los resultados de este estudio, la técnica de instrumentación mecanizada se mostró más eficaz en la reducción del tiempo y en el grado de llenado de los canales radiculares. El uso de la instrumentación mecanizada proporcionó ventajas en la rutina clínica pediátrica. 


\section{Referencias bibliográficas}

1. Farhin K, Vamsi KC, Manohar P, Shilpa S, Bhavesh R. Comparison of Cleaning Efficacy and Instrument Time between Rotary and Manual Instrumentation Techniques in Primary Teeth: An in vitro Study. Int J of Clin Pediatr Dent. 2016; 9: 124-27.

2. Mello-Moura ACV, Borelli T, Matos R, Bonini GC, Moura-Netto C. Como podemos otimizar a endodontia em dentes decíduos? Relato de caso. Rev Assoc Paul Cir Dente. 2013; 67: 50-5.

3. Souza JM, Leite VV, Resende GB, Rocha MJC: Obturação tridimensional dos canais radiculares. lentulo ou lima endodôntica? Rev Odonto Ciência - Fac. Odonto/PUCRS. 2005; 20 (47)

4. Rosa, MF, Modesto A, Faraco, IMJ. Manual and Rotary Instrumentation Techniques for Root Canal Preparation in Primary Molars. Dentistry 3000, 2014; 2(1) 2167-8677.

5. Guines AS, Faria G, Tanomaru-Filho M, Bonetti-Filho. Influence of Sealer Placement Technique on the Quality of Root Canal Filling by Lateral Compaction or Single Cone. Braz Dent J. 2014; 25: 117- 22.

6. Kummer TR, Evaluation of Removal From Manual and Rotary Instrumentation Techniques in Human primary Teeth -in vitro study. [Dissertação] - Rev Pós Grad em Odont, Universidade Federal de Santa Catarina, Florianópolis. 2006: 146f.

7. Mello-Moura ACV, Cerqueira DF, Santos EM. Pasta Guedes-Pinto - Revisão de Literatura: 26 anos de estudos sobre citotoxidade, citotóxicos, histopatológicos, microbiológicos e clínicos. Rev Pós Grand. 2007; 14: 260-6.

8. Waterhouse PJ. Nunn JH. Whitworth JM. Soames JV. Primary Molar Pulp Therapy - Histological Evaluation of Failure. Int J Paediatr Dent. 2000; 10: 313-21.

9. Alves FRF, Rôças IN, Almeida BM, Neves MAS, Zoffoli J, Siqueira Jr F. Quantitative Molecular and Culture Analyses of Bacterial Elimination in Oval-Shaped Root Canals by a Single-File Instrumentation Technique. Int Endod J. 2012; 10: 65-91.

10. Andrade APRCBA, Silva SREP, Imparato JCP, et al. Viabilidade de Dentes Decíduos Artificiais para Mensuração Eletrônica do Comprimento de Trabalho dos Canais Radiculares. Pesq Bras Odontoped Clín Integr. 2013; 13: 83-87.

11. Guedes-Pinto AS, Santos EM. Tratamento endodôntico em dentes decíduos. In: Guedes-Pinto AC. Odontopediatria. 8 ed. São Paulo: Santos, 2010.

12. Pereira HSC, Silva EJNL, Coutinho Filho TS. Movimento reciprocante em Endodontia: revisão de literatura. Rev Bras Odontol. 2012; 69,: 246-9.

13. Silva LA, Leonardo MR, Nelson-Filho P, Tanomaru JM. Comparison of Rotary and Manual Instrumentation Techniques on Cleaning Capacity and Instrumentation Time in Deciduous Molars. J Dent Child 2004; 71: 45-7. 14. Kummer TR, Calvo MC, Cordeiro MM, de Sousa Vieira R,Carvalho Rocha MJ. Ex Vivo Study of Manual and Rotary Instrumentation Techniques in Human Primary Teeth. Oral Surg Oral Med Oral Pathol Oral RadiolEndod. 2008; 105: 84-92.

15. Nagaratina PJ, Shashikirann D, Subbareddy VV. In Vitro Comparison of NiTi Rotary Instruments in Root Canal Preparations of Primary and Permanent Molar. J Indian Soc Pedog Prev Dent. 2006; 24 : $186-91$.

16. Barr ES, Kleier DJ, Barr NV. Use of Nickel-Titanium Rotary Files for Root Canal Preparation in Primary Teeth. Pediatr Dent 1999; 21: 453-454.

17. Pinheiro HHC, Assunção LRS, Torres DKB, Miyahara LAN, Arantes DC. Terapia Endodôntica em Dentes Decíduos por Odontopediatras. Pesq Bras Odontoped Clin Integr. 2013; 13: 351-60. 
Lopes Reis M, Coelho PM, Carvalho M, Vieira Silva R, Quadros Ravazzi TP.

18. Kramer PF, Faraco Junior IM, Feldens CA. Current Status of Pulp Therapy in the Brazilian Universities Pulpotomy and Pulpectomy Technique in Deciduous Teeth. J Bras Odontoped e Odonto Bebê. 2000; 3: $222-9$. 19. Brusco EHS, Perussolo B, Scapin HLC, Ferreira SLM. Procedimentos e substâncias Empregadas por Faculdades de odontologia Brasileiras na Terapia Endodôntica de Dentes Decíduos Pulpectomizados. J Bras Odonto pediatr Odonto Bebê. 2002; 5:35-46.

20. Guedes-Pinto AC. Odontopediatria, Santos Editora 2012; 8:593-8.

Recibido: 05/10/2017

Aceptado: 02/12/2017

Correspondencia: Monique Lopes Reis.nick_loppes@hotmail.com. 